\title{
A study of convection velocities in a zero pressure gradient turbulent boundary layer
}

\author{
J. LeHew, M. Guala \& B. J. McKeon*
}

\begin{abstract}
Time-resolved DPIV measurements performed in wall parallel planes at several wall normal locations in a turbulent boundary layer (TBL) are used to illuminate the distribution of wall parallel velocities in a three-dimensional energy spectrum over streamwise, spanwise, and temporal wavelengths. To our knowledge, this is the first time this type of spectral distribution has been reported. Slices of the 3D spectrum can give insight into the propagation of different scales in the flow as well as the streamwise and spanwise extent of dominant scales. Measurements were performed at three wall normal locations, $y^{+}=34,108$, and 278 , in a zero pressure gradient TBL at $R e_{\tau}=470$. Two high speed cameras placed side-by-side in the streamwise direction give a $10 \delta$ streamwise field of view with a time step of $\Delta t^{+}=0.5$ between consecutive fields. Far from the wall the convection velocities of all scales are very close to the local mean velocity in agreement with the work of Dennis and Nickels, ${ }^{\mathbf{1}}$ while at $y^{+}=34$ it was found that all measured scales in the flow convect faster than the local mean in agreement with Krogstad et. al. ${ }^{2}$ The variation of the convection velocity with scale and distance from the wall will be discussed.
\end{abstract}

\section{Nomenclature}

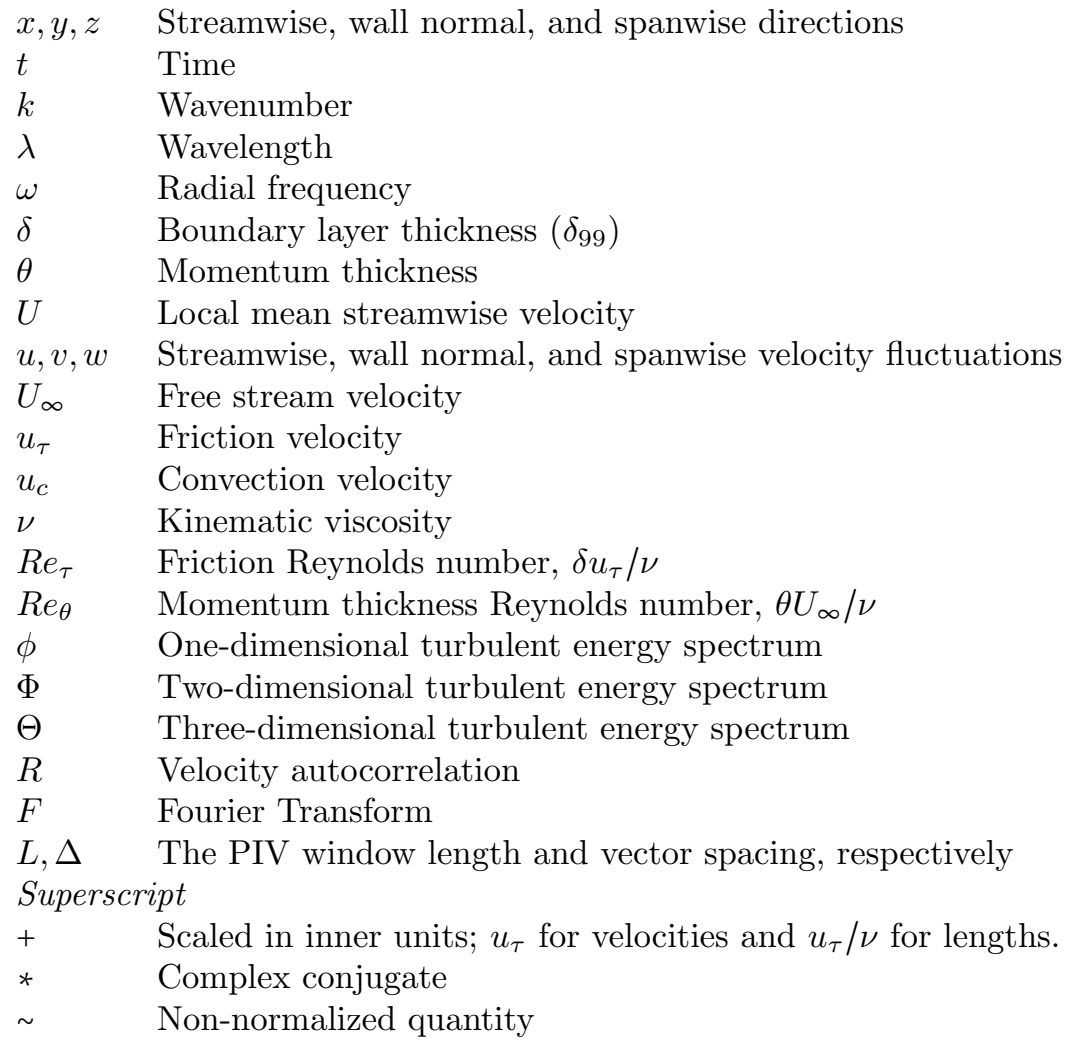

${ }^{*}$ Graduate Aerospace Laboratories, California Institute of Technology, Pasadena, CA 91125, U.S.A. 


\section{Introduction}

Our current understanding of the structure of the turbulent boundary layer over a large range of Reynolds number rests primarily upon spatial turbulence spectra calculated from low Reynolds number direct numerical simulations and temporal spectra in high Reynolds number experiments, where the accurate conversion from the temporal to the spatial representation and vice versa is an important fundamental concern. While temporal information can be extracted from DNS data, the resources required to analyze a time resolved flow over a sufficiently long time period would be enormous. Similarly, in experiments, most commonly used instruments, such as hot wire anemometers, provide temporal information at a single point, and thus a spatial representation of the flow requires multiple arduous measurements at two or more points simultaneously. A conversion between the spatial and temporal domain can be done using Taylor's frozen turbulence hypothesis ${ }^{3}$ which states that the spatial field can be reconstructed from the temporal field if the convection velocities of the individual eddies/scales which compose the flow are known. This conversion is done assuming these eddies are "frozen", or in other words, that their shape does not evolve significantly over the distance projected. It is often assumed that the convection velocity of all such eddies is the local mean velocity at the point of measurement, yet many studies have shown that this is not always a valid assumption depending on the wall normal location or scales considered. For a recent example, the study of Monty and $\mathrm{Chong}^{4}$ has shown that the differences in the spectral distribution between temporal measurements in experiments and spatial measurements in computations of the same flow under the same conditions can be partially rectified by using a scale dependent convection velocity near the wall.

There have been several studies performed to determine the convection velocities in various canonical boundary layer flows. The experiments of Morrison, et.al. ${ }^{5}$ used two-dimensional frequency-wavenumber spectra to study the convection velocities of scales in the viscous sublayer of a pipe, which were discovered to travel at a fixed velocity for the entire sublayer. They also presented data outside of the sublayer $\left(y^{+}=15\right)$ where it is apparent that the large scales at this location are traveling faster than the local mean. The channel DNS of Kim and Hussain ${ }^{6}$ and the boundary layer experiments of Krogstad, et.al. ${ }^{2}$ both found a deviation in the convection velocity away from the local mean near the wall as well as a variation of this velocity with the scales considered. In particular, Krogstad found that the smallest scales in the flow consistently travel slower than the local mean at all wall normal locations, and that large scales travel faster than the local mean as far from the wall as $y^{+} \approx 40$. The channel flow computations of del Álamo and Jiménez ${ }^{7}$ show similar deviations near the wall using a different method for computing the convection velocity. This method provides the variation of the convection velocity with both streamwise and spanwise scale. In the channel LES of Chung and McKeon ${ }^{8}$ it was additionally found that large scales travel slightly slower than the local mean in the wake region of the boundary layer (i.e. very far from the wall).

Recent time-resolved DPIV investigations by Dennis and Nickels ${ }^{1}$ at $y / \delta=0.16$ with a $6 \delta \times 3 \delta$ field of view demonstrated the validity of applying Taylor's hypothesis to project temporal information at least $6 \delta$ downstream, and also demonstrated the validity of using the local mean as the convection velocity at this particular wall normal location. In the present study time-resolved DPIV will also be used, but in a larger domain $(10 \delta \times 5 \delta)$ where regions closer to the wall $(y / \delta=0.07)$ and further from the wall $(y / \delta=0.59)$ will also be investigated. The focus will be on the spectrum as opposed to the spatial fields. The differences discovered at all wall normal locations will be explained and their implications discussed.

\section{Experimental Setup}

Experiments were performed over a $1 \mathrm{~m}$ long by $0.45 \mathrm{~m}$ wide Plexiglass flat plate situated in a $2 \mathrm{~m}$ long by $1 \mathrm{~m}$ wide test section in a free surface water tunnel facility. The boundary layer on the bottom of the plate was studied to avoid interaction with surface waves. A shroud with a wedge-shaped leading edge was placed around the spanwise edges of the plate to promote a 2-dimensional flow. The leading edge of the plate was a 1:8 ellipse which merged smoothly with the rest of the plate. A thin strip of tape (approximately $3 \mathrm{~mm}$ long, $0.25 \mathrm{~mm}$ thick, and spanning the width of the plate) was placed immediately downstream of the elliptical leading edge on the measurement side to promote transition. A wedge shaped flap was attached at the trailing edge to control the location of the stagnation point. The plate was suspended by four threaded rods with which the angle of the plate in both the streamwise and spanwise direction could be controlled. The flow was conditioned by passing it through a perforated plate, a honey comb, three turbulence reducing screens and finally a 6:1 contraction. Measurements were made with a free stream velocity of $0.67 \mathrm{~m} / \mathrm{s}$ starting at 
a location $0.63 \mathrm{~m}$ downstream of the leading edge. A diagram of the tunnel and a photograph of the test section are shown in Figure 1.

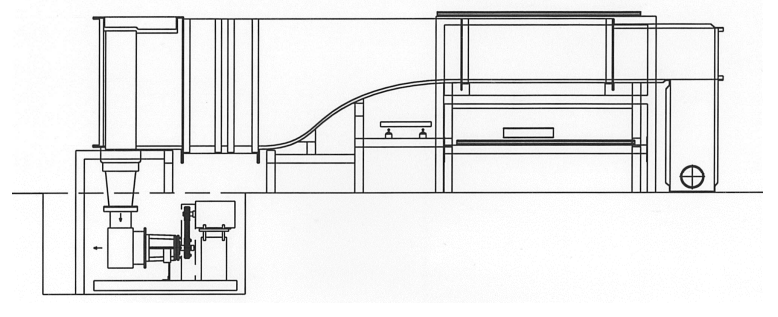

(a) Tunnel Diagram ${ }^{9}$

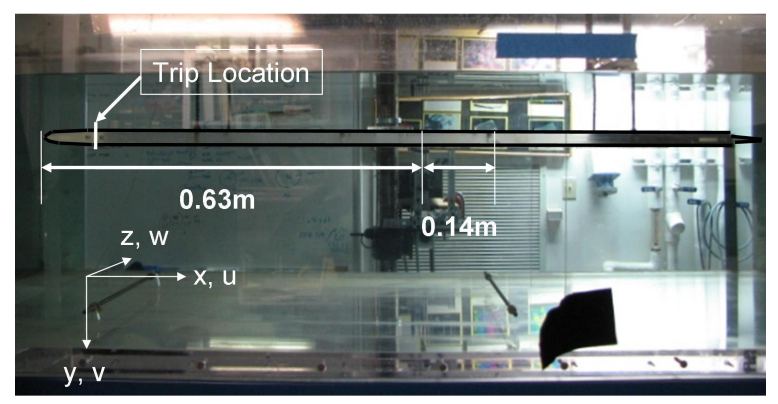

(b) Test Section

Figure 1. Diagram of the experimental apparatus

Time resolved 2D DPIV was used to measure the flow both normal and parallel to the flat plate using a LaVision PIV system with 2 Photron Fastcam APX-RS high speed cameras operating at 2000fps with $1024 \times 1024$ pixel resolution. The flow is seeded with $6 \mu \mathrm{m}$ glass beads and illuminated by a Photonics DM20527 solid state laser.

Wall normal measurements were taken first to characterize the flow at the start of the measurement location $(0.63 \mathrm{~m}$ downstream) using only one camera. The field of view was $50.6 \mathrm{~mm}$ in the streamwise and wall normal directions. A laser sheet was guided into the test section from below to illuminate the flow field. Due to camera memory limitations, a maximum of 2048 images could be taken at a time. For collapse of the mean profile and mean square turbulent fluctuations, 5 experiments were performed and the results were averaged together. The data was processed using LaVision's Davis software with multipass processing from $16 \times 16$ to $12 \times 12$ pixel windows with $50 \%$ overlap, and the first window was centered at $y^{+}=8$. The non-dimensional vector spacing $\left(\Delta y^{+}\right)$and time step $\left(\Delta t^{+}\right)$are presented in Table 1.

For the wall parallel measurements, 2 cameras were placed side-by-side to extend the field of view in the streamwise direction and capture large scale motions. The cameras were mounted to an optics table under the test section and viewed the bottom of the plate via a mirror. The cameras were adjusted to create some overlap between the two image planes. The field of view was approximately $140 \mathrm{~mm}$ in the streamwise direction and $70 \mathrm{~mm}$ in the spanwise direction with some slight changes between the three planes investigated. The laser sheet, approximately $1 \mathrm{~mm}$ thick in the measurement plane, was passed through the side of the test section and was centered at locations $1 \mathrm{~mm}, 3.2 \mathrm{~mm}$, and $8.2 \mathrm{~mm}$ from the wall in order to capture the near wall, outer log layer, and wake regions of the turbulent boundary layer. At each measurement location, 40 experiments were performed for over 80,000 images per plane. The data was processed using $32 \times 32$ pixel windows with $50 \%$ overlap. The non-dimensional field size $\left(L_{x}, L_{z}\right)$, vector spacing $\left(\Delta x^{+}, \Delta z^{+}\right)$, and wall normal locations $\left(y^{+}\right)$are presented in Table 1.

\section{Calculations and data normalization}

The mean velocity profile and boundary layer thickness are calculated from measurements in the wall normal plane averaged in the streamwise direction (the boundary layer thickness varied by only $5 \%$ over the entire measurement window). The mean profile is shown in Figure 2(a) where the mean velocity for each wall parallel measurement is denoted by a square symbol. The boundary layer thickness and Reynolds number are reported in Table 1.

Since no direct measurements of the wall shear stress were made in this experiment, the friction velocity was calculated from a least squares fit to the predetermined log layer marked with circles in Figure 2(a). Comparisons were also made to the experiments of DeGraaff and Eaton ${ }^{10}$ at a similar Reynolds number $\left(\operatorname{Re}_{\theta}=1430\right)$. The first two wall normal points $\left(y^{+}=8,18\right.$, not shown $)$ have been removed due to their deviation from this profile. Additional flow parameters are summarized in Table 1. From here on, the focus will be on measurements made in wall parallel planes. 
Table 1. Parameters for the experiment

\begin{tabular}{ccccccccc}
\hline$R e_{\tau}$ & $R e_{\theta}$ & $\delta$ & $\theta$ & $U_{\infty}$ & $u_{\tau}$ & $\nu / u_{\tau}$ & $\Delta y^{+}$ & $\Delta t^{+}$ \\
\hline 470 & 1280 & $13.9 \mathrm{~mm}$ & $1.72 \mathrm{~mm}$ & $0.67 \mathrm{~m} / \mathrm{s}$ & $0.03 \mathrm{~m} / \mathrm{s}$ & $29.5 \mu m$ & 10.0 & 0.5 \\
\hline \multicolumn{1}{c}{} & & & & & & & \\
\hline Plane & $y^{+}$ & $y / \delta$ & $U^{+}$ & $u_{r m s}^{+}$ & $w_{r m s}^{+}$ & $\Delta x^{+}, \Delta z^{+}$ & $L_{x} / \delta$ & $L_{z} / \delta$ \\
\hline 1 & 34 & 0.07 & 13.1 & 2.10 & 0.88 & 37.6 & 9.98 & 4.91 \\
2 & 108 & 0.23 & 16.4 & 1.53 & 0.91 & 37.1 & 9.85 & 4.84 \\
3 & 278 & 0.59 & 20.2 & 1.25 & 0.78 & 37.5 & 10.03 & 4.89 \\
\hline
\end{tabular}

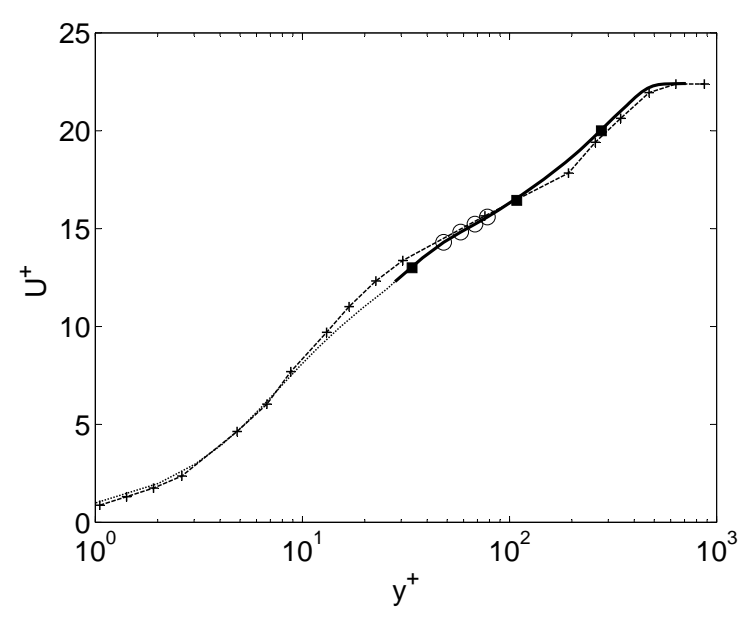

(a) Mean Profile

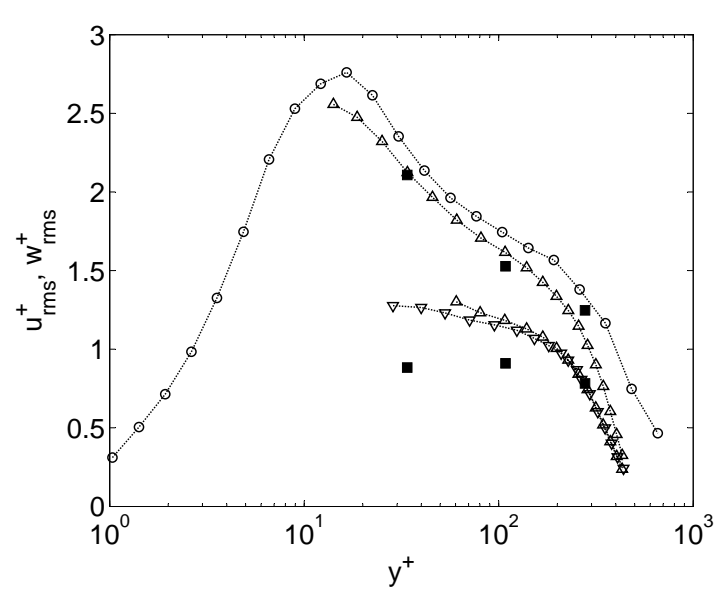

(b) Streamwise and Spanwise Fluctuations

Figure 2. In both figures, a represents the wall parallel data. In (a) The mean velocity profile is presented where the symbols are - : Mean profile from wall normal measurements, $\cdots$ : Spalding fit, ${ }^{11}{ }_{-}-+--$: data from DeGraaff and Eaton ${ }^{\mathbf{1 0}}$ at $\operatorname{Re}_{\theta}=1430$, and $\bigcirc$ : points selected to represent the log layer. In (b) the wall parallel rms velocity fluctuations are presented where the symbols are: - - -- : data from Degraaff and Eaton ${ }^{10}$ at $R e_{\theta}=1430,--\triangle--$ : data from Erm and Joubert ${ }^{12}$ at $\operatorname{Re}_{\theta}=1003$, and $--\nabla--$ : data from Erm and Joubert ${ }^{12}$ at $\operatorname{Re}_{\theta}=1568$.

The rms velocity fluctuations from each wall parallel plane are presented in Figure 2(b) alongside the data from DeGraaff and Eaton. ${ }^{10}$ Both streamwise and spanwise fluctuations are underestimated for wall normal locations $y^{+}<200$. There are two sources for this underestimation.

First, due to the finite thickness of the laser sheet (estimated to be 35 plus units in extent), the wall parallel measurements were averaged over a range of wall normal locations. This averaging biases the correlation used in the calculation of the velocity vectors toward slower moving particles and this bias is enhanced by the large velocity gradients in the near wall region. ${ }^{13}$ Thus, the averaging will reduce the measured fluctuation level slightly, and this level will decrease as the velocity gradient increases near the wall. This effect is seen in Figure 2(b) for both the spanwise and streamwise fluctuations.

The second source of underestimation of the turbulent fluctuations comes from the attenuation of scales below a certain size. As discussed by Foucaut et.al., ${ }^{14}$ scales less than 2.25 times the window size will be attenuated, with the attenuation increasing as the length scale decreases, independently of the window overlap. As shown in Figure 3, this cutoff can attenuate a significant portion of the spectrum which is identical to an attenuation of the mean square fluctuations. This affects a much larger portion of the spanwise velocity spectrum since its energy is spread over a higher wavenumber range. Thus, this attenuation affects the spanwise fluctuations more severely than the streamwise ones, which is evident when comparing the underestimation of $w_{r m s}$ to that of $u_{r m s}$ in Figure 2(b). In this case, the balance between a large interrogation window for improved accuracy and a small window for better resolution is important. 


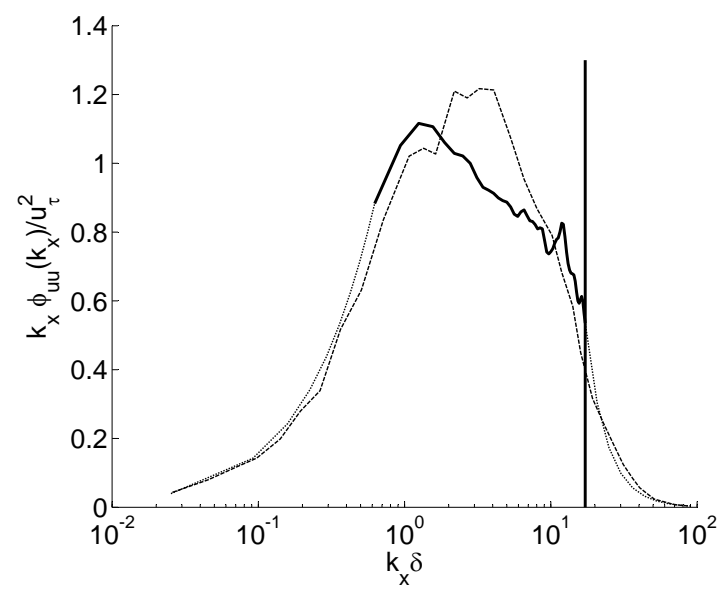

(a) Streamwise Spectrum

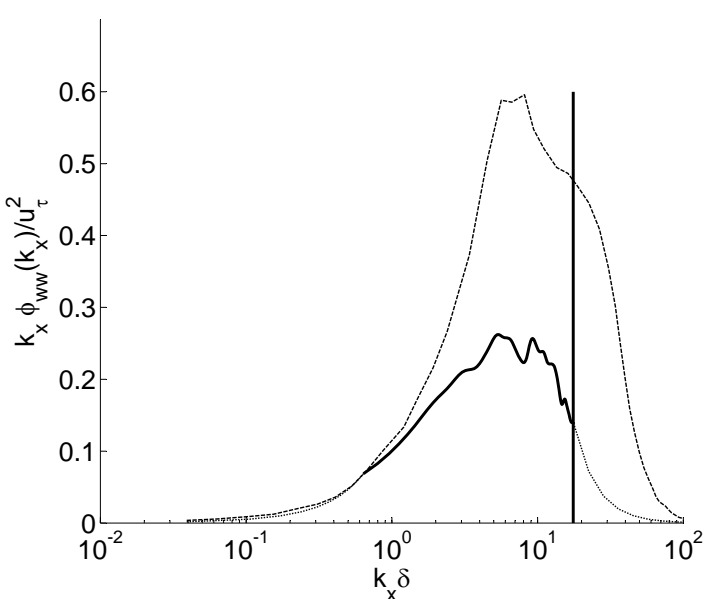

(b) Spanwise Spectrum

Figure 3. Streamwise and spanwise spectra over streamwise wavenumbers for $y / \delta=0.07$. The spectra are denoted by the solid lines with estimated tails dotted. They are normalized by $\overline{u^{2}}$ and $\overline{w^{2}}$, respectively, which explains the low peak in (b). The dashed lines are spectra from Erm and Joubert ${ }^{12}$ at $y / \delta=0.1$ and the vertical solid line is the cutoff wavenumber described by Foucaut et.al. ${ }^{14}$

For calculation of the spectral density, the most general definition is given in Equations 1 and 2 relating the three-dimensional spectral density, $\Theta$, to the autocorrelation, $\mathrm{R}$, where $\mathrm{R}$ is defined in equation 3 for streamwise fluctuations.

$$
\begin{aligned}
& \Theta_{u u}\left(k_{x}, k_{z}, \omega\right)=\frac{1}{(2 \pi)^{3}} \iint_{-\infty}^{\infty} \int_{u u} R_{u}\left(\rho_{x}, \rho_{z}, \tau\right) \exp \left(-i\left(\rho_{x} k_{x}+\rho_{z} k_{z}+\tau \omega\right)\right) d \rho_{x} d \rho_{z} d \tau \\
& R_{u u}\left(\rho_{x}, \rho_{z}, \tau\right)=\iint_{-\infty}^{\infty} \int_{u u}\left(k_{x}, k_{z}, \omega\right) \exp \left(i\left(\rho_{x} k_{x}+\rho_{z} k_{z}+\tau \omega\right)\right) d k_{x} d k_{z} d \omega \\
& R_{u u}\left(\rho_{x}, \rho_{z}, \tau\right)=\frac{\overline{\overline{u(x, z, t) u\left(x+\rho_{x}, z+\rho_{z}, t+\tau\right)}}}{\sqrt{\overline{\overline{u^{2}(x, z, t)}}} \sqrt{\overline{u^{2}\left(x+\rho_{x}, z+\rho_{z}, t+\tau\right)}}}
\end{aligned}
$$

As already shown, the spanwise spectrum is not well resolved due to the window size needed for accuracy (32x32 pixels) so the focus will be on the streamwise velocity spectra and the direction subscripts (e.g. "uu") will be omitted henceforth. Using the cross-correlation theorem the calculation of the spectral density reduces to finding the Fourier Transform of the velocity fluctuations given in Equation 4 where the tilde indicates that the spectrum is not yet normalized.

$$
\tilde{\Theta}\left(k_{x}, k_{z}, \omega\right)=F(u(x, z, t)) F^{\star}(u(x, z, t))
$$

The spectrum is normalized such that:

$$
\overline{u^{2}}=\int_{0}^{k_{x, \max }} \int_{0}^{k_{z, \max }} \int_{0}^{\omega_{\max }} \Theta\left(k_{x}, k_{z}, \omega\right) d k_{x} d k_{z} d \omega
$$

In the above, the upper limits are set by the vector spacing and the lower limits are zero. If the full data field is to be split into subsets (e.g. dividing the time history into multiple overlapping subsets for improved convergence), care must be taken not to deteriorate the resolution near the peak of the spectrum. A normalized 3D spectrum for $y / \delta=0.23$ is presented in Figure 4 to aid in understanding this energy distribution. Here the data has been split into 3 subsets in time and 2 subsets in the spanwise direction with $50 \%$ overlap which produced no negative effects on the spectral distribution.

The common 1D and 2D spectra can be calculated using a lower order version of Equation 4 and averaging over several times and/or positions. These times and positions are chosen to be far enough apart to be statistically independent, and thus the average performed is identical to averaging over separate experiments. 
Note that there is no averaging done in the streamwise direction. The calculation of the $2 \mathrm{D}$ spectrum $\tilde{\Phi}\left(k_{x}, k_{z}\right)$ is shown in equation 6 as an example. The $1 \mathrm{D}$ calculation is similar.

$$
\tilde{\Phi}\left(k_{x}, k_{z}\right)=\frac{1}{N_{t}} \sum_{n=1}^{N_{t}} F\left(u\left(x, z, t_{n}\right)\right) F^{*}\left(u\left(x, z, t_{n}\right)\right)
$$

In Equation 6, the times at which the velocity field is sampled, $t_{n}$, are chosen for statistical independence, as stated previously, and the spectrum is normalized by $\overline{u^{2}}$ as in Equation 5 .

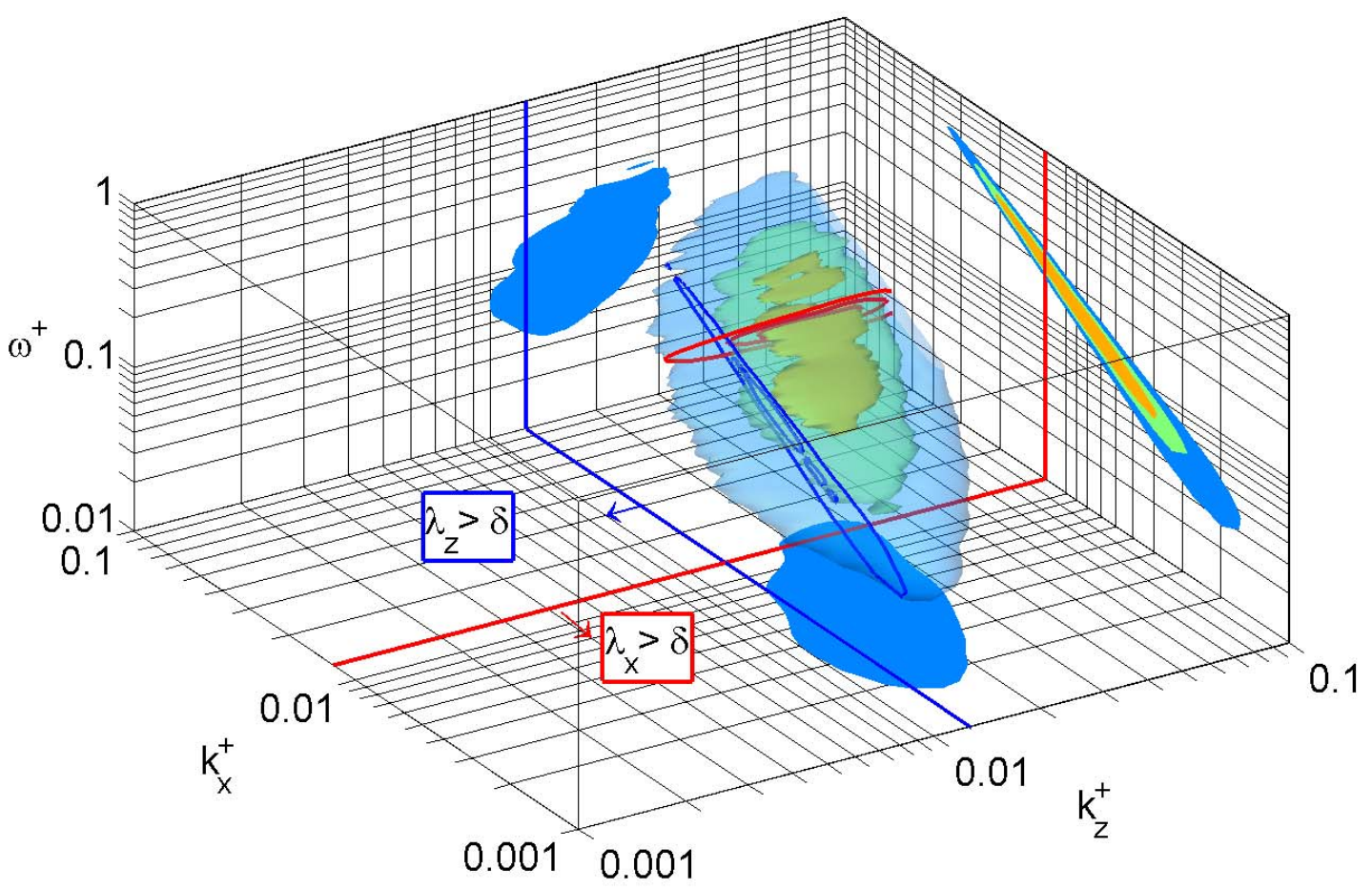

Figure 4. The premultiplied 3D spectrum, $k_{x} k_{z} \omega \Theta\left(k_{x}, k_{z}, \omega\right)$, at $y / \delta=0.23$ is represented by isosurfaces whose energy levels are 25, 50, and $70 \%$ of the maximum energy. The premultiplied integrations of the spectrum, $k_{x} k_{z} \Phi_{3 D}\left(k_{x}, k_{z}\right)$, etc., are presented on the respective axes where the colors indicate the same energy levels; these are not simply projections of the 3D spectrum. The solid red and blue lines denote the region where scales are larger than the boundary layer thickness in streamwise and spanwise extent. These regions are also shown as cuts in the $3 \mathrm{D}$ spectrum.

Alternatively, the $1 \mathrm{D}$ and 2D spectra can be calculated by integrating the 2D and 3D spectra, respectively. Equation 7 gives the integral calculation of the same 2D spectrum as in Equation 6. All of the premultiplied integrated spectra are shown on the respective axes in Figure 4. Again, the 1D calculation is similar.

$$
\Phi_{3 D}\left(k_{x}, k_{z}\right)=\int_{0}^{\omega_{\max }} \Theta\left(k_{x}, k_{z}, \omega\right) d \omega
$$

The subscript "3D" indicates that the spectrum was calculated by integration of the $3 \mathrm{D}$ spectrum. This spectrum is already normalized since $\Theta$ is normalized (see Equation 5). Also, note that the integral in the definition should ideally be from 0 to $\infty$, but, of course, only a portion of the spectrum is captured by our measurements.

Ideally, $\Phi_{3 D}$ and the $\Phi$ should be the same, but due to limited wavenumber and frequency ranges in the direction of integration, the two will differ to some degree over some or all of the wavenumber/frequency space. In addition, the attenuation at high wavenumbers may complicate the picture. The same is true when integrating $2 \mathrm{D}$ spectra to recover $1 \mathrm{D}$ spectra. Thus, care must be taken when interpreting any integrated spectrum. 


\section{Results}

The 3D spectrum shown in Figure 4 is a concise way of illustrating the distribution of energy in wall parallel planes and could be an important source of information when considering temporal perturbations to the boundary layer such as the response of the boundary layer to a control input. The spectral distributions for all planes are similar where the energy is concentrated around a line in the $\left(k_{x}, \omega\right)$ plane and more diffuse in the $\left(k_{x}, k_{z}\right)$ plane as can be deduced from the 2D spectra. Most of the energy lies in scales that are narrower than the boundary layer thickness in the spanwise direction, yet larger than the boundary layer thickness in the streamwise direction, consistent with recent findings.

To study the convection velocities of particular scales in the flow, a number of methods have been proposed in the literature. Most of the classical methods are based on analyzing the premultiplied $\Phi\left(k_{x}, \omega\right)$ spectrum, or equivalently, the time-space correlation, and have been reviewed by Goldschmidt, et.al. ${ }^{15}$ The methods include looking for: a maximum in the temporal spectrum at each streamwise separation, a maximum in the streamwise spectrum at each frequency, or a line of maxima along $k_{x} \omega \Phi\left(k_{x}, \omega\right)$, all of which can give different results. ${ }^{15}$ Here we will consider the third method. In Figure $5, k_{x} \omega \Phi\left(k_{x}, \omega\right)$ is presented for each wall parallel plane with the local mean velocity indicated by a solid black line. By looking at the spectral ridge (line of maxima), it is clear that at $y / \delta=0.23$ all scales travel near the local mean and the use of the local mean velocity as the convection velocity is valid in agreement with the findings of Dennis and Nickels ${ }^{1}$ at a similar wall normal location $\left(y / \delta=0.16\right.$ and $\left.R_{\theta}=4685\right)$. Near the wall, though, it appears that all resolved scales travel faster than the local mean. This agrees with the findings of Krogstad et.al. ${ }^{2}$ at a similar location and Reynolds number $\left(y^{+}=38.4\right.$ and $\left.R e_{\theta}=1409\right)$. Their convection velocity data is included in Figure 5(a) for comparison. Finally, the third plane presents a slight deviation at very low wavenumbers where the scales appear to travel slightly slower than the local mean as noted in the LES of Chung and McKeon. $^{8}$

The spread of the spectral energy in all plots in Figure 5 as well as the variation of the ridge location can be explained with the aid of Figure 6 . Here we present a simplified model where three small eddies, $S$, $S_{H}$, and $S_{L}$, with wavenumber $k_{S}$, and one large eddy, $L$, with a wavenumber $k_{L}$ intersect the measurement location $y_{1}$ where the local velocity is $U_{1}$. While eddies $\mathrm{S}$ and $\mathrm{L}$ are centered on the measurement location, the eddy $S_{H}$ is centered on a region with a higher mean velocity and the eddy $S_{L}$ on a region with a lower mean velocity (hence the subscript $H$ stands for high velocity and $L$ for low velocity). The eddies should convect at a velocity equal to the local mean velocity of the locations on which they are centered. Knowing this convection/phase velocity, the frequency associated with an eddy of a particular size can be found using the definition of the convection velocity: $u_{c}=\omega / k_{x}$. Thus eddies $\mathrm{S}$ and $\mathrm{L}$ will lie along the line $\omega / k_{x}=U_{1}$, while eddy $S_{H}$ will be above this line and the eddy $S_{L}$ below, as shown on the right hand side of Figure 6 . In terms of the energy contributed, eddies $S$ and $L$ will contribute significant amounts of energy since they are centered on this measurement plane, while the other two eddies will contribute lesser amounts. This variation of energetic contributions from eddies above and below the measurement location helps to explain the spread of the spectrum seen in all of plots in Figure 5.

The deviations of the spectral ridge can be explained using the same model. It was shown that for a measurement location very near the wall (as in Figure 5(a)), the spectrum will lose its symmetry about the mean and the spectral ridge will move above the local mean line. This loss of symmetry is caused by the wall blocking effect which prevents large eddies, such as eddy L, form being centered on planes near the wall, such as the plane on which eddy $S_{L}$ lies. Thus all energetic contributions from low wavenumbers (large wavelengths) must come from eddies located on planes further from the wall. Noting that eddies on the order of the boundary layer thickness likely have their centers near the center of the boundary layer, their energetic imprint at the wall will be moving faster than the local mean and will promote the movement of the spectral ridge above the local mean line. The opposite effect can be expected above the centerline of the boundary layer (as in Figure 5(c)), where the imprint of these large eddies will be traveling slightly slower than the local mean.

These findings imply that one must be very careful in interpreting single point measurements very near and far from the wall as the projected structural picture may be distorted by not only a change in the mean convection velocity, but also a convection velocity which is scale dependent. Further analysis of the convection velocity is planned using the convection velocity definition presented by del Álamo and Jiménez ${ }^{7}$ which provides a convection velocity for each streamwise and spanwise scale pair given the 3D spectrum calculated here. This could be particularly useful in describing the structure of the turbulent boundary layer and determining the dominant scales in the flow. In addition, it will also be important to look at the 


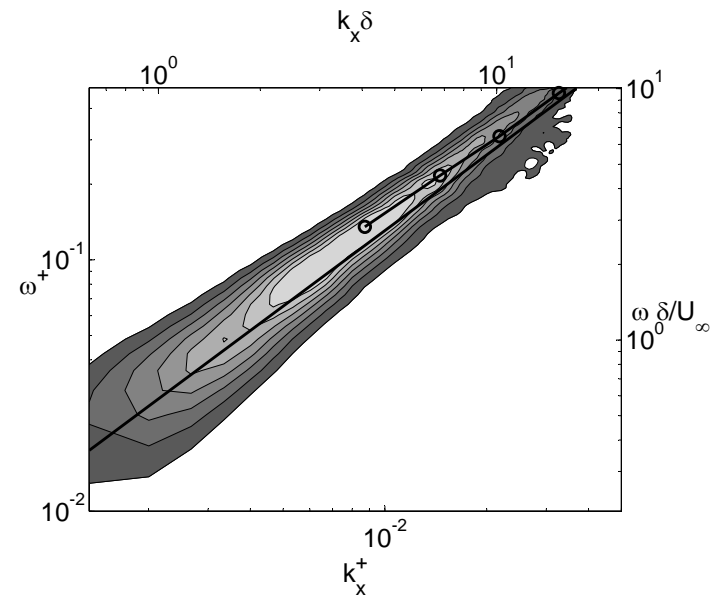

(a) Plane 1

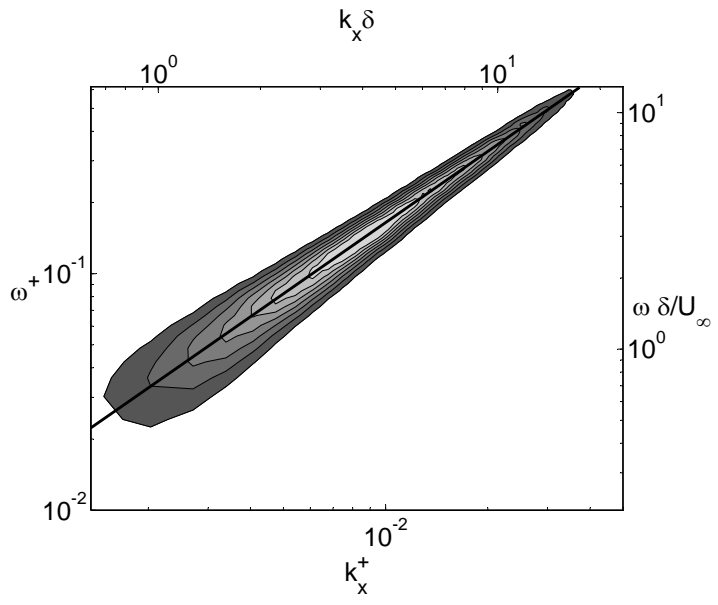

(b) Plane 2

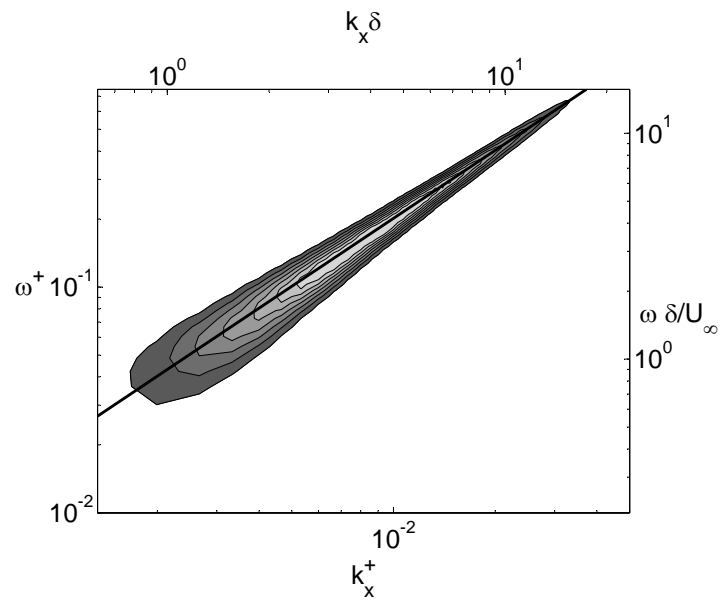

(c) Plane 3

Figure 5. In all figures above, the contours represent 20 to $80 \%$ of the maximum energy of the premultiplied spectrum $k_{x} \omega \Phi\left(k_{x}, \omega\right)$ in $\mathbf{1 0 \%}$ increments. - : Local mean velocity, - $\bigcirc-$ : Convection velocities from Krogstad et.al. ${ }^{2}$ at $y^{+}=38.4$ and $\operatorname{Re}_{\theta}=1409$.
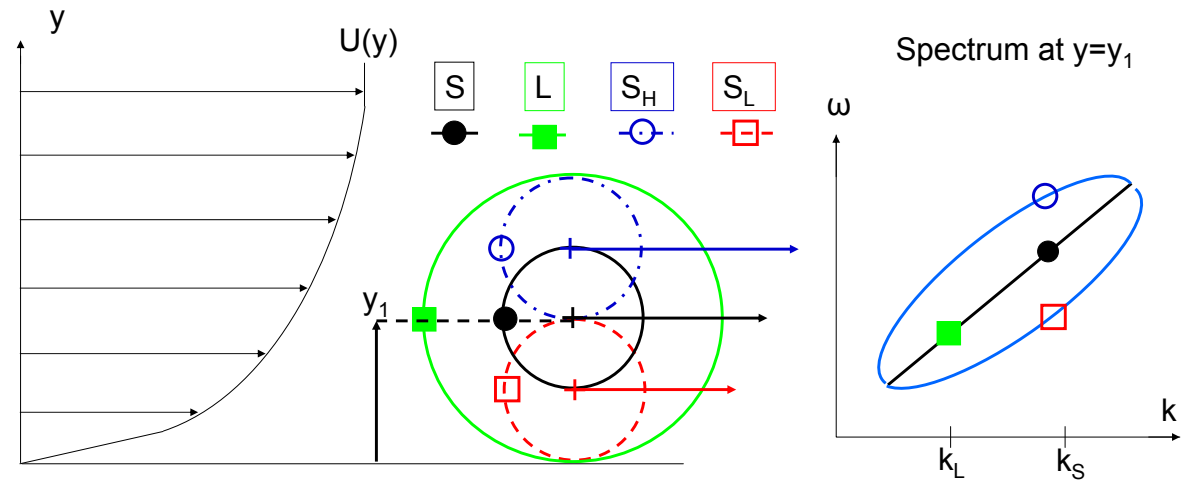

Figure 6. A cartoon explaining the variation of convection velocity with distance from the wall 
spatial fields and see what parallels can be drawn between the spectral representation and the structural representation of the flow. In particular, how the projection of a temporal measurement and the actual spatial field compare with and without a correct choice of the convection velocity would be of interest.

\section{Conclusions}

From the experiments presented, the distribution of turbulent energy over streamwise, spanwise, and temporal wavelengths and the convection velocity of these scales at different wall normal locations were investigated using time-resolved DPIV. The lower than expected turbulent statistics found near the wall in wall parallel measurements were explained to be the result of averaging over the laser sheet thickness. In addition, the attenuation of the turbulent fluctuations for scales less that 2.25 times the window size was recognized as a major source of error in the calculation of both the spanwise fluctuations and spectra, and thus the focus was on streamwise fluctuations where this effect was minimal. Understanding these limitations, a 3-D spectrum was presented showing, for the first time, the distribution of energy over streamwise, spanwise, and temporal scales. 2D spectra over streamwise wavenumber and frequency were used to study the convection velocity in each wall parallel plane. In the plane nearest the wall, $y / \delta=0.07$, it was found that all scales traveled faster than the local mean while for the plane furthest from the wall, the largest scales in the flow traveled slower than the local mean. These deviations were explained by the use of a simple model. Future work will be focused on extending the analysis to the distribution of convection velocities for scales of particular streamwise and spanwise extent, and the analysis of the measured velocity fields to draw relations between the spectral and structural representations of the turbulent boundary layer.

\section{Acknowledgments}

The support provided for this project by the Air Force Office of Scientific Research (program manager John Schmisseur) under award \#FA9550-09-1-0701 is gratefully acknowledged.

\section{References}

\footnotetext{
${ }^{1}$ Dennis, D. J. C. and Nickels, T. B., "On the limitations of Taylor's hypothesis in constructing long structures in a turbulent boundary layer," J. Fluid Mech., Vol. 614, 2008, pp. 197-206.

${ }^{2}$ Krogstad, P. A., Kaspersen, J. H., and Rimestad, S., "Convection velocities in a turbulent boundary layer," Phys. Fluids, Vol. 10, No. 4, 1998, pp. 949-957.

${ }^{3}$ Taylor, G. I., "The spectrum of turbulence," Proc. R. Soc. Lond. A, Vol. 164, 1938, pp. 476-490.

${ }^{4}$ Monty, J. P. and Chong, M. S., "Turbulent channel flow: comparison of streamwise velocity data from experiments and direct numerical simulation," J. Fluid Mech., Vol. 633, 2009, pp. 461-474.

${ }^{5}$ Morrison, W. R. B., Bullock, K. J., and Kronauer, R. E., "Experimental evidence of waves in the sublayer," J. Fluid Mech., Vol. 47, 1971, pp. 639-656.

${ }^{6}$ Kim, J. and Hussain, F., "Propagation velocity of perturbations in turbulent channel flow," Phys. Fluids A, Vol. 5, No. 3, 1993, pp. 695-706.

${ }^{7}$ del Álamo, J. C. and Jiménez, J., "Estimation of turbulent convection velocities and corections to Taylor's approximation," J. Fluid Mech., Vol. 640, 2009, pp. 5-26.

${ }^{8}$ Chung, D. and McKeon, B. J., "Large-eddy simulation of large-scale structures in long channel flow," J. Fluid Mech., 2010, In Press.

${ }^{9}$ Bobba, K. M., Robust Flow Stability: Theory, Computations and Experiments in Near Wall Turbulence, Ph.D. thesis, California Institute of Technology, 2004.

${ }^{10}$ DeGraaff, D. B. and Eaton, J. K., "Reynolds-number scaling of the flat-plate turbulent boundary layer," J. Fluid Mech, Vol. 422, 2000, pp. 319-346.

${ }^{11}$ Spalding, D. B., "A single formula for the law of the wall," Trans. ASME E, Vol. 28, 1961, pp. 455-458.

${ }^{12}$ Erm, L. P. and Joubert, P. N., "Low-Reynolds-number turbulent boundary layers," J. Fluid Mech., Vol. 230, 1991, pp. $1-44$.

${ }^{13}$ Westerweel, J., "Fundamentals of digital particle image velocimetry," Meas. Sci. Technol., Vol. 8, 1997, pp. $1379-1392$.

${ }^{14}$ Foucaut, J. M., Carlier, J., and Stanislas, M., "PIV optimization for the study of turbulent flow using spectral analysis," Meas. Sci. and Tech., Vol. 15, 2004, pp. 1046-1058.

${ }^{15}$ Goldschmidt, V., Young, M., and Ott, E., "Turbulent convective velocities (broadband and wavnumber dependent) in a plane jet," J. Fluid Mech., Vol. 105, 1981, pp. 327-345.
} 